\title{
The Relationship of Adiponectin Level with Non- Alcoholic Fatty Liver Disease (NAFLD) Degrees
}

\author{
Annisa Dwi Andriani ${ }^{1}$, Gontar Alamsyah Siregar ${ }^{2}$, Dharma Lindarto ${ }^{3}$, Taufik \\ Sungkar ${ }^{2}$ \\ ${ }^{1}$ Department of Internal Medicine, Faculty of Medicine, Universitas Sumatera Utara, Medan \\ ${ }^{2}$ Division of Gastroentero-hepatology, Department of Internal Medicine, Faculty of Medicine, Universitas \\ Sumatera Utara, Medan \\ ${ }^{3}$ Division of Metabolic and Endocrinology, Department of Internal Medicine, Faculty of Medicine, Universitas \\ Sumatera Utara, Medan
}

Corresponding Author: Gontar Alamsyah Siregar

\begin{abstract}
Background: NAFLD is liver fat that exceeds $5 \%$ of the total liver weight. Adiponectin is thought to have a role in the development of insulin resistance and is associated with the incidence of NAFLD. This study aims to find the relationship between adiponectin levels and the degree of NAFLD.
\end{abstract}

Methods: Sixty seven study subjects with metabolic syndrome went through abdominal ultrasound to diagnose NAFLD and measure BMI, serum adiponectin levels, and NAFLD Fibrosis Score (NFS). The relationship between adiponectin levels, degrees of NAFLD, NFS, and comorbid would be assessed.

Results: Forty one (61\%) was male and 26 $(38.8 \%)$ were female with a mean age of 42.08 years. From the abdominal ultrasound, the number of NAFLD grade I, II, and III were $40.4 \%, 29.8 \%, 29.8 \%$. The mean adiponectin levels in all samples were 7.13 (3.25-11.45) $\mu \mathrm{g} / \mathrm{ml}$. Both type 2 diabetes and BMI $\geq 23$ $\mathrm{kg} / \mathrm{m}^{2}$ were associated with the degree of NAFLD ( $p<0.001)$. In type 2 diabetes and BMI $\geq 23 \mathrm{~kg} / \mathrm{m}^{2}$ group, the mean adiponectin level was found to be lower ( $\mathrm{p}<0.001)$. The relationship between NFS and NAFLD, also adiponectin levels and the degree of NFS was significant $(\mathrm{p}<0.001)$.

Conclusion: The relationship between adiponectin levels with the degree of NAFLD and NFS were statistically significant.

Keywords: NAFLD, NFS, adiponectin, fibrosis, metabolic syndrome

\section{INTRODUCTION}

Non-Alcoholic Fatty Liver Disease (NAFLD) is a condition resulting from the accumulation of excessive fat in the form of triglycerides (steatosis) in the liver of more than $5 \%$ of all hepatocytes. ${ }^{[1]}$ Its prevalence is increasing rapidly worldwide in parallel with the increase in obesity and type 2 diabetes. ${ }^{[2]}$ The prevalence of NAFLD in the general population is estimated to be 20$30 \%$ in Western countries and $15 \%$ in Asian countries. In Indonesia, not much data is obtained on the prevalence or incidence of NAFLD. In a study in the city of Jakarta, the prevalence of NAFLD was $30 \%$, most in middle age $(37.2 \%)$. NAFLD is enforced with two components: i) the presence of evidence of fatty liver and or steatohepatitis histologically or by imaging and ii) exclusion of other causes of fatty liver, such as significant alcohol consumption, use of drugs that are steatogenic or hereditary disorders. $^{[3]}$

A single biochemical marker to identify NAFLD has yet to be found. In the laboratory image of NAFLD patients, there was a slight increase in aminotransaminase enzyme levels, even about $78 \%$ of NAFLD patients had normal liver enzymes. Another laboratory feature that can be seen in NAFLD is an abnormality of the lipid profile. Previous studies have shown an increase in triglyceride levels, low density 
lipoprotein (LDL), total cholesterol and a decrease in high density lipoprotein (HDL) levels. Adiponectin is thought to have a role in the occurrence of conditions of insulin resistance and is associated with the incidence of NAFLD. ${ }^{[4]}$ Adiponectin is important in NAFLD, in several studies it was found that NAFLD patients had lower levels of Adiponectin. ${ }^{[4]}$

Based on literary tracing conducted by the author, there are still many differences in the results obtained from the relationship between adiponectin levels and the degree of NAFLD. In Indonesia, no previous research has been published looking at the relationship between adiponectin levels and the degree of NAFLD. This study aims to find the relationship between adiponectin levels and the degree of NAFLD.

\section{MATERIAL AND METHODS}

The research design was cross sectional and conducted at the Integrated Diagnostic Installation RSUP H. Adam Medan, North Sumatra after obtaining approval from the Health Research Ethics Commission. The research was conducted from January 2020 to August 2020. Sixty seven people with NAFLD who met the inclusion criteria and did not meet the exclusion criteria who were taken by consecutive sampling. Inclusion criteria were: age $\geq 18$ years, patients diagnosed with NAFLD from history taking, laboratory examination and abdominal ultrasound, received written and voluntary informed consent. Exclusion criteria were: suffering from hepatitis B or hepatitis C, malignancy or autoimmune disease, alcohol consumption $>30 \mathrm{~g} /$ day in men and $>20$ $\mathrm{g} /$ day in women. There are 4 things needed for the diagnosis of NAFLD according to the European Association for the Study of the Liver (EASL): the presence of hepatic steatosis from histological or radiological examination, no significant alcohol consumption, no other concurrent etiology and no other causes of chronic liver disease.
Anamnesis was carried out regarding previous medical history and history of alcohol consumption. Abdominal ultrasound in this study was chosen to diagnose NAFLD. Abdominal ultrasound examination is a non-invasive test to diagnose NAFLD. Abdominal ultrasound examination is carried out by one operator who has the competence in ultrasound examination to confirm the NAFLD image. If the results of the examination are inconclusive, then the diagnosis of NAFLD is carried out by 2 competent operators. The appearance of NAFLD on ultrasound is at least two of the following findings: increased hepatorenal contrast, bright liver texture, deep beam attenuation, vascular blurring and gall bladder wall definition. Then the degree of NAFLD is determined as: I) the echogenicity of the liver is diffusely increased but the periportal and diaphragm are still quite good, II) diffuse increase in liver echogenicity obscures periportal echogenicity but diaphragmatic echogenicity is still quite good, III) diffuse increase in liver echogenicity obscures the periportal as well as the echogenicity of the diaphragm. Furthermore, measurement of height and weight to evaluate BMI, then laboratory tests to check serum adiponectin levels, fasting blood glucose, platelet count, albumin, aminotransferase enzyme level. The results of these tests were then entered into the NAFLD Fibrosis Score (NFS) formula to classify the predicted liver fibrosis.

Data analysis of the relationship between adiponectin levels and the degree of NAFLD, the relationship between NFS and the degree of NAFLD and the relationship between adiponectin levels and NFS used the ANOVA test if the data were normally distributed, and used the KruskalWallis test if the data were not normally distributed. The relationship between comorbid and the degree of NAFLD used the chi-square hypothesis test if the data were normally distributed; if not normal then Fisher's exact test was used. As for the comorbid relationship with adiponectin 
levels, the Independent T-test will be used if the data is normally distributed and will be replaced with the Mann-Whitney test if the data is not normally distributed. The analysis result is significant if the $\mathrm{p}$ value $<0.05$.

\section{RESULTS}

This study was followed by 67 patients who met the inclusion criteria, 41 (61\%) were male and 26 patients $(38.8 \%)$ were women with a mean age of 42.08 years. The most comorbid found was type 2 diabetes. From the results of abdominal ultrasound, it was found that the highest number was grade I NAFLD (40.4\%). The mean adiponectin levels in all samples were $7.13(3.25-11.45) \mu \mathrm{g} / \mathrm{ml}$.

Table 1. Demographic and clinical characteristics

\begin{tabular}{|l|l|}
\hline Characteristics & $\mathbf{n}=\mathbf{6 7}$ \\
\hline Gender & $41(61,2)$ \\
- Men & $26(38,8)$ \\
- Women & $42,08(25-65)$ \\
\hline Age, mean (min-max) & \\
\hline Ethnic & $37(55,2)$ \\
- Bataknese & $24(35,8)$ \\
- Javanese & $6(9)$ \\
- Acehnese & \\
\hline Employment & $30(44,8)$ \\
- Private employees & $15(22,4)$ \\
- Housewives & $17(25,4)$ \\
- Entrepreneur & $5(7,5)$ \\
- Government employees & \\
\hline Education & $3(4,5)$ \\
- Elementary & $15(22,4)$ \\
- Junior high school & $31(46,3)$ \\
- Senior high school & $18(26,9)$ \\
\hline - Bachelor & \\
\hline Comorbid & $37(55,2)$ \\
\hline Type 2 Diabetes Mellitus & $34(50,7)$ \\
\hline BMI $\geq 23 \mathrm{~kg} / \mathrm{m}^{2}$ & $15(22,4)$ \\
\hline Hypertension & \\
\hline NAFLD grading: & $27(40,4 \%)$ \\
\hline NAFLD grade I & $20(29,8 \%)$ \\
\hline NAFLD grade II & $20(29,8 \%)$ \\
\hline NAFLD grade III & $7.13(3.25-11.45)$ \\
\hline Mean adiponectin levels $(\mu \mathrm{g} / \mathrm{ml})$ & \\
\hline
\end{tabular}

\section{Relationship between Adiponectin Levels and NAFLD Degrees}

By using the Kruskal-Wallis test, it was found that there was a relationship between adiponectin levels and the degree of NAFLD, with a statistically significant result ( $\mathrm{p}<0.01)$. The mean serum adiponectin levels in the NAFLD grade I, II, and III groups were 9.59 (8.03-11.45) $\mu \mathrm{g} / \mathrm{ml}, 6.82$ (6.16-7.95) $\mu \mathrm{g} / \mathrm{ml}, 4.12$ (3.254.98) $\mu \mathrm{g} / \mathrm{ml}$.

Table 2. The Relationship between Adiponectin Levels and NAFLD Severity

\begin{tabular}{|l|l|l|}
\hline & Adiponectin $(\boldsymbol{\mu g} / \mathbf{m l})$ & $\boldsymbol{p}$ value \\
\cline { 1 - 2 } & \multirow{2}{*}{\begin{tabular}{l}
\multirow{2}{*}{ NAFLD grade I } \\
NAFLD grade II
\end{tabular}} & \\
\cline { 1 - 2 } NAFLD grade III & $6.82(8.03-11.45)$ & \\
\cline { 1 - 2 } & $4.12(3.16-7.95)$ & \\
\hline
\end{tabular}

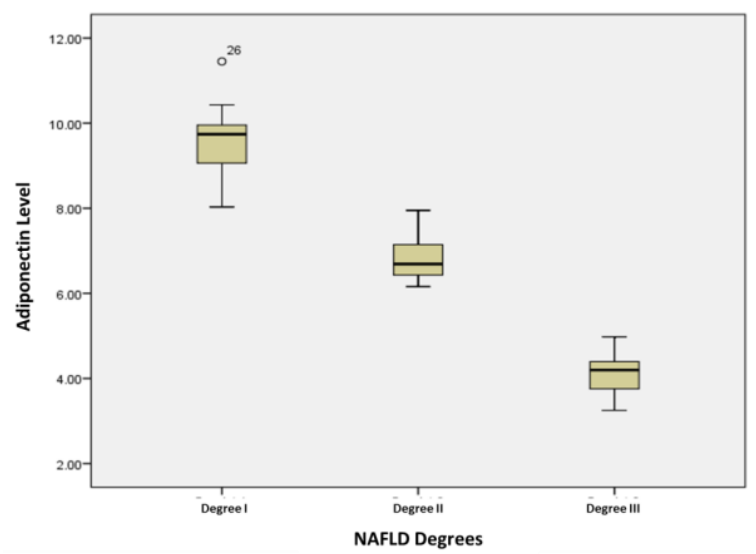

Figure 1. Relationship of Adiponectin Levels and NAFLD severity

\section{Correlation between Comorbid and NAFLD and Adiponectin Levels}

Comorbid are type 2 diabetes, BMI $\geq 23 \mathrm{~kg} / \mathrm{m} 2$, and hypertension. Both type 2 diabetes and $\mathrm{BMI} \geq 23 \mathrm{~kg} / \mathrm{m} 2$, at the time of the Fisher-exact test, both had a significant relationship with $\mathrm{p}$-value $<0.001$, while the hypertension comorbidity was not significant $(\mathrm{p}=0.93)$.

Table 3. Relationship of comorbid and NAFLD severity

\begin{tabular}{|c|c|c|c|c|c|}
\hline Comorbid & NAFLD grade I (\%) & NAFLD grade II (\%) & NAFLD grade III (\%) & Total & p value \\
\hline \multicolumn{6}{|l|}{ Type 2 DM } \\
\hline Yes & $6(8.9)$ & $14(20.8)$ & $17(25)$ & $37(55.2)$ & \multirow[t]{2}{*}{$<0.001^{*}$} \\
\hline No & $21(31.3)$ & $6(8.9)$ & $3(4.4)$ & $30(44.8)$ & \\
\hline \multicolumn{6}{|c|}{ BMI $\geq 23 \mathrm{~kg} / \mathrm{m}^{2}$} \\
\hline Yes & $5(7.4)$ & 13(19.4) & $16(23.8)$ & $34(50.8)$ & \multirow[t]{2}{*}{$<0.001 *$} \\
\hline No & $22(32.8)$ & $7(10.4)$ & $4(5.9)$ & $33(49.2)$ & \\
\hline \multicolumn{6}{|c|}{ Hypertension } \\
\hline Yes & $5(7.4)$ & $4(5.9)$ & $6(8.9)$ & $15(22.3)$ & \multirow[t]{2}{*}{0.93} \\
\hline No & $22(32.8)$ & $16(23.8)$ & $14(20.8)$ & $52(77.7)$ & \\
\hline
\end{tabular}

\footnotetext{
*Fisher Exact test
} 
In the sample with comorbid type 2 $\mathrm{DM}$ and the group with $\mathrm{BMI} \geq 23 \mathrm{~kg} / \mathrm{m} 2$, the mean adiponectin level was found to be lower, and with the Mann-Whitney test both had a significant relationship with p-value $<0.001$. Whereas in comorbid hypertension, blood pressure > $140 \mathrm{mmHg}$ had a lower mean adiponectin level, and it was not significant $(\mathrm{p}=0.696)$.

Table 4. Relationship of comorbid and Adiponectin Levels

\begin{tabular}{|l|l|l|}
\hline Comorbid & $\begin{array}{l}\text { Mean adiponectin level }(\boldsymbol{\mu g} / \mathbf{m l}) \\
(\text { min-max) }\end{array}$ & p value \\
\hline DM type 2 & $6.03(3.25-10.40)$ & $<0,001$ \\
\hline Yes & $8.48(3.61-11.45)$ & \\
\hline No & $5.94(3.25-10.40)$ & $<0,001$ \\
\hline BMI & $8.35(3.61-11.45)$ & \\
\hline $23 \mathrm{~kg} / \mathrm{m}^{2}$ & 0,696 \\
\hline Hypertension & $6.80(3.25-11.45)$ & \\
\hline$>140 \mathrm{mmHg}$ & $7.22(3.53-10.40)$ & \\
$<140 \mathrm{mmHg}$ &
\end{tabular}

\section{Relationship between NFS and NAFLD Degrees}

The lowest average NFS was in the first grade NAFLD group, namely -1,966 (4.293 - 3.190), the NFS mean at grade II NAFLD was $-0.422(-4.326-1.189)$ and grade III was $1.323(-1.926-3.524)$, then tested with Kruskal Wallis, both of them had a significant relationship ( $p<0.001)$.

Table 5. NFS Rating Parameters
\begin{tabular}{|l|l|}
\hline Parameter & $24.02(19.32-33.59)$ \\
\hline Mean BMI (min-max), $\mathrm{kg} / \mathrm{m} 2$ & $202(98-380)$ \\
\hline $\begin{array}{l}\text { Mean random blood glucose (min-max) } \\
(\mathrm{mg} / \mathrm{dl})\end{array}$ & $1,4(0.87-2.20)$ \\
\hline Mean AST/ALT ratio (min-max) & $220(89-398)$ \\
\hline Mean thrombocyte (min-max), $\mathrm{x} 10^{3} / \mathrm{mm}^{3}$ & $3,3(2.5-4.6)$ \\
\hline Mean albumin (min-max), $\mathrm{mg} / \mathrm{dl}$ & $7.13(3.25-11.45)$ \\
\hline Median adiponectin, $(\mathrm{min}-\mathrm{maks}), \mu \mathrm{g} / \mathrm{ml}$ & \\
\hline
\end{tabular}

Table 6. Relationship of NFS and NAFLD Severity

\begin{tabular}{|l|l|l|}
\hline NAFLD & Mean NFS (min-max) & p value \\
\hline Grade I & $-1.966(-4.293-3.190)$ & $<0,001$ \\
\cline { 1 - 2 } Grade II & $-0.422(-4.326-1.189)$ & \\
\cline { 1 - 2 } Grade III & $1.323(-1.926-3.524)$ & \\
\hline
\end{tabular}

\section{Relationship between Adiponectin Levels and NFS}

By using the Kruskal-Wallis test, it was found that there was a relationship between adiponectin levels and the degree of NFS with a statistically significant result (p-value $<0.001$ ).
Table 1. Relationship between adiponectin level with NFS Score

\begin{tabular}{|l|l|l|}
\hline & $\begin{array}{l}\text { Adiponectin, median, } \\
\boldsymbol{\mu g} / \mathbf{m L}\end{array}$ & $\begin{array}{l}\boldsymbol{p} \\
\text { value }\end{array}$ \\
\hline Low $(<-1,445)$ & $8.78(4.46-11.45)$ & $<0,001$ \\
\cline { 1 - 2 } Intermediate $(-1,445-0,676)$ & $7.02(3.61-10.43)$ & \\
\hline Advanced $(>0,676)$ & $4.89(3.25-10.40)$ & \\
\hline
\end{tabular}

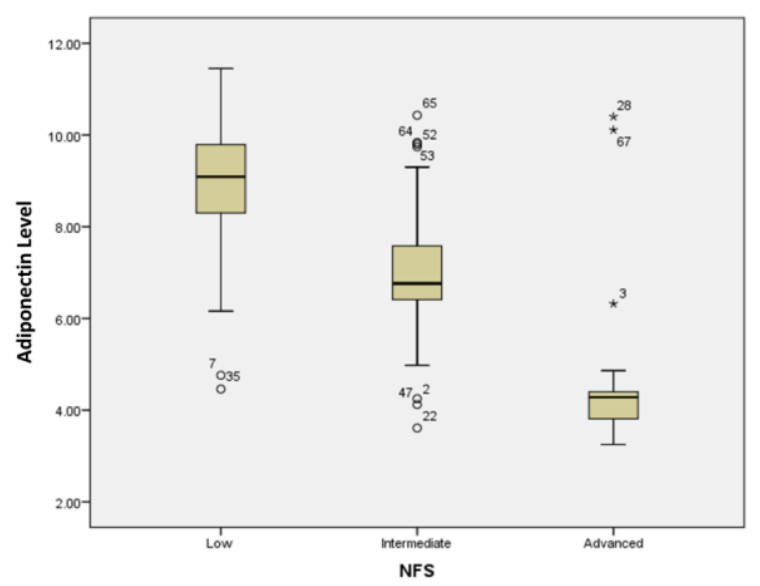

Figure 2. Adiponectin level in three different NFS score grade

\section{DISCUSSION}

The degree of NAFLD in this study has a relationship with adiponectin levels with a statistically significant result ( $p$ $<0.001$ ). Several other studies which found that adiponectin levels were lower in the NAFLD group than in the control group have been summarized in a meta-analysis study including 28 studies on 2243 subjects (698 controls, 1545 NAFLD patients) conducted by Polyzos et al in 2011, in that study using liver biopsy to diagnose NAFLD. For example, in a study conducted by Argentou et al in 2009, adiponectin in NAFLD $(4.36 \pm 2.24 \mu \mathrm{g} / \mathrm{ml})$ was lower than in control group $(5.70 \pm 1.31 \mu \mathrm{g} / \mathrm{ml}) .{ }^{[5]}$ Another study by Bugianesi in 2005, lower adiponectin levels found in NAFLD (5.52 \pm $2.2 \mu \mathrm{g} / \mathrm{ml})$ compared to controls $(10.7 \pm$ $5.89 \mu \mathrm{g} / \mathrm{ml}) .{ }^{[6]}$ Study by Haukeland in 2006, serum adiponectin levels in NAFLD $(5.6 \pm 2.9 \mu \mathrm{g} / \mathrm{ml})$ were lower than controls $(12.0 \pm 5.3 \mu \mathrm{g} / \mathrm{ml}) .{ }^{[7]}$ And there are still several other studies that support the results of this study. The conclusion from this meta-analysis study is that early examination of adiponectin levels in NAFLD patients can predict the course of severe disease such as liver fibrosis and hepatocellular carcinoma, so that further research is expected to be carried out for 
adiponectin therapy. The more severe the severity of the NAFLD process into fibrosis and then liver cirrhosis, the lower the adiponectin levels obtained from this study. ${ }^{[8]}$ Some previous data show adiponectin has a protective role in liver damage in NAFLD. ${ }^{9}$

Contrary, in a study conducted by Balmer in 2010, it was found that adiponectin levels were increased in patients with cirrhosis of the liver, adiponectin levels were in the same direction as the liver fibrosis process, but still adiponectin levels fell in NAFLD patients. Adiponectin levels in cirrhotic patients $18.6 \pm 14.5 \mu \mathrm{g} / \mathrm{ml}$ compared to the non-cirrhotic group $8.4 \pm$ $6.1 \mu \mathrm{g} / \mathrm{ml}$ with adiponectin control 9.1 $\mu \mathrm{g} / \mathrm{ml}$, in the NALFD group adiponectin levels were $4.8 \pm 3 . .5 \mu \mathrm{g} / \mathrm{ml}$. The weakness of this study does not mention the specific etiology of cirrhosis. ${ }^{[10]}$ Likewise, the results of Silva et al's study in 2017, adiponectin levels were higher in the cirrhosis group $(21.59 \mu \mathrm{g} / \mathrm{ml})$ compared to the control group $(12.52 \mu \mathrm{g} / \mathrm{ml})$ with $\mathrm{p}$ value $<0.001$. This research is a cohort study with a sample size of 122 people. In the discussion of the study, it was said that patients with liver cirrhosis have a broad-spectrum factor from metabolic disease, the interactions between these factors such as insulin resistance, cholesterol levels, nutritional status, are still not clearly known. ${ }^{[11]}$

In this study, it was found that comorbid in patients with NAFLD were type 2 diabetes, $\mathrm{BMI} \geq 23$, and hypertension. Previous research conducted by Pagano et al in 2005 found that low adiponectin levels in patients with NAFLD were closely related to insulin resistance, metabolic syndrome, type 2 diabetes, and hypertension. ${ }^{[9]}$ It is known that there is a strong relationship between insulin resistance and NAFLD. Insulin resistance causes fat accumulation characterized by increased levels of free fatty acid (breakdown of fat tissue), increased fatty acids and triglyceride synthesis in the liver. Insulin resistance and hyperinsulinemia cause an inflammatory process and a fibrosis process to take place in the liver. ${ }^{[12]}$ The liver is the main target of adiponectin, where the liver contains adiponectin receptors (AdipoR1 and AdipoR2). Adiponectin binding and its receptors will stimulate phosphorylation of AMPK, PPAR $\alpha$, and fatty acid oxidation in the liver. All of these mechanisms are inhibited by resistin. Low levels of adiponectin in people with NAFLD cause fat accumulation in the liver. Meanwhile, high levels of adiponectin are reported to prevent NAFLD by reducing fatty acid synthesis due to inhibition of acyl-CoA carboxylase (ACC) and fatty acid synthesis. Decreased ACC activity causes decreased levels of malonyl-CaA (inhibits Carnitine Palmitoyltransferas I / CPT-I) and oxidation of fatty acids. ${ }^{[13]}$

NAFLD patients with comorbid DM in this study were found as many as 37 people $(55.2 \%)$ from all samples and had a significant relationship with $\mathrm{p}<0.001$. In a meta-analysis study conducted by Younossi et al in 2016 on 729 studies with 8,515,431 samples from 22 countries, it was found that the prevalence of type 2 diabetes sufferers with NAFLD was $22.51 \%$. ${ }^{[14]}$ Type 2 diabetes has a very close relationship with NAFLD, where more than $75 \%$ of people with type 2 diabetes are reported to have NAFLD. In type 2 diabetes, insulin resistance increases lipolysis of adipose tissue which causes the release of free fatty acids and deposited in the liver then becomes steatosis. Type 2 diabetes is a significant risk factor for progression to liver fibrosis and cirrhosis. [14] The association of comorbid diabetes mellitus type 2 with serum adiponectin levels was found to have a lower mean adiponectin level with $\mathrm{p}<0.001$. These results are consistent with a meta-analysis study conducted by Wang et al in 2018 in China, average adiponectin level was lower in diabetes patients $\left[\begin{array}{llll}6.7 & (5.2-8.3) & \mu \mathrm{g} / \mathrm{ml}\end{array}\right]$ compared to the control group [8.4 (6.510.8) $\mu \mathrm{g} / \mathrm{ml}]$ with $\mathrm{p}$-value $<0.001$. In this study, it was concluded that there was a close relationship between type 2 diabetes and adiponectin levels in the Chinese 
population, so that the adiponectin level examination was considered for targeting adiponectin therapy, diet, and physical activity at high risk of type 2 diabetes. ${ }^{[15]}$

NAFLD sufferers with a BMI $\geq 23$ $\mathrm{kg} / \mathrm{m}^{2}$ were found in 34 people $(50.8 \%)$ from all samples, with the most being 16 people $(23.8 \%)$ with grade III NAFLD, then 13 people $(19.4 \%)$ at grade II NAFLD, and at least 5 people $(7.4 \%)$ on grade I NAFLD with $\mathrm{p}<0.001$. The association between BMI $\geq 23 \mathrm{~kg} / \mathrm{m}^{2}$ and serum adiponectin levels was found to be lower in mean adiponectin levels. In the 2016 Younossi study globally, it was found that the prevalence of obese patients with NAFLD was $51.34 \% .{ }^{[14]}$ Previous studies reported that visceral adipose significantly increased the risk of NAFLD, NASH and fibrosis. In a previous cohort study showing an association between weight change and the incidence of NAFLD, a light weight gain of $2 \mathrm{~kg}$ over normal body weight based on BMI was shown to increase the risk of NAFLD. Obesity causes two times the risk of developing steatosis in the liver. ${ }^{[15]}$

In this study, there were 15 people with NAFLD with comorbid hypertension (22.3\%, $\mathrm{p}=0.93)$. In a previous study conducted by Younossi et al in 2016, it was found that the prevalence of comorbid hypertension in NAFLD was $39.34 \% .{ }^{[16]}$ In a study conducted by Oikonomou et al in 2018, hypertension and NAFLD were found to be related. NAFLD is associated with new-onset hypertension, whereas increased blood pressure is associated with the progression of fatty liver to liver fibrosis. Activation of the renin-angiotensin aldosterone system (RAAS) has a role in NAFLD, so the administration of RAAS antagonists is beneficial in NAFLD patients. ${ }^{[17]}$ The hypertension group with serum adiponectin did not have a significant relationship ( $p=0.685)$. In a meta-analysis study conducted by Kim et al in 2013 in Boston, identifying 43 non prospective and 5 prospective studies with 17,598 adult samples, found that serum adiponectin levels in hypertensive patients were 1.64 $\mu \mathrm{g} / \mathrm{ml}[95 \%$ CI: (- 2.07)). ) - (- 1.21)] than the normal group. Each increase in serum adiponectin levels by $1 \mu \mathrm{g} / \mathrm{ml}$ reduces the risk of hypertension by $6 \%$ (95\% CI: $0.92-$ 0.97). ${ }^{[18]}$

The NAFLD Fibrosis Score (NFS) is one of the most accurate scoring systems for identifying advanced fibrosis among patients with NAFLD. In the research of Angulo et al. 2007, it was found that NFS is a fairly good scoring system with a sensitivity of $77 \%$ with a specificity of $77 \%$. In this study, 17 people $(25.3 \%)$ had low results, 25 indeterminate $(37.3 \%)$, and advanced 32 people $(47.7 \%){ }^{[19]}$ Recently, the American Association for the Study of Liver Diseases (AASLD) guidelines for NAFLD management recommended NFS as a screening tool to identify advanced fibrosis. ${ }^{[3]}$ Compared with other composite scores for advanced fibrosis, NFS was found to perform well. ${ }^{[20]}$ Of all the parameters in this study, the calculation of the NAFLD Fibrosis Score (NFS) was carried out with the formula $1.657+0.037 \mathrm{x}$ age (years) $+0.094 \times$ BMI $(\mathrm{kg} / \mathrm{m} 2)+1.13 \mathrm{x}$ impaired fasting blood glucose/diabetes $($ there $=1$, none $=0)+0.99 \times$ AST/ALT ratio -0.013 x Platelet (x $109 / \mathrm{L})-0.66 \mathrm{x}$ albumin $(\mathrm{g} / \mathrm{dl})$, then the score calculation results are classified into: Low $(<-1.445)$, Intermediate (-1.4465-0.676), and Advanced (>0.676). From the results of the Fisherexact test, it was found that there was a relationship between NFS and the degree of NAFLD with $\mathrm{p}$ value $<0.001$. Cross tabulation was performed between NFS and NAFLD degrees and obtained the lowest NFS mean in the first degree NAFLD group $[-1,966(-4,293-3,190)]$, the mean NFS at NAFLD grade II was $-0.422 \quad(-4.326$ 1.189). and the highest NFS was at NAFLD grade III $[1,323(-1,926-3,524)]$. By Kruskal Wallis test, both of them have a significant relationship with a $\mathrm{p}$ value $<0.001$. Furthermore, it was found that there was a relationship between adiponectin levels and the degree of NFS with a statistically significant result $(\mathrm{p}<0.001)$. 
This study still had a lot of limitation. It is necessary to carry out further studies with a better study design such as a prospective cohort to assess the relationship of adiponectin levels with NAFLD grade and NAFLD Fibrosis Score, so that the relationship can be known more definitely.

\section{CONCLUSIONS}

There was a statistically significant relationship between adiponectin levels and the degree of NAFLD and also adiponectin levels and the degree of NAFLD Fibrosis Score $(\mathrm{p}<0.001)$.

\section{ACKNOWLEDGEMENTS}

The authors gratefully acknowledge that the present research is supported by the Ministry of Research and Technology and Higher Education Republic of Indonesia. The support is under the research grant DRPM (Direktorat Riset dan Pengabdian Masyarakat).

\section{Conflict of Interest: None}

\section{Source of Funding: None}

\section{Ethical Approval: Approved}

\section{REFERENCES}

1. LaBrecque D, Abbas Z, Anania F, Ferenci P, Khan AG, Goh KL, et al. World Gastroenterology Organisation Global Guidelines. Nonalcoholic fatty liver disease and nonalcoholic steatohepatitis. World Gastroenterology Organisation.2014

2. Satapathy SK, Sanyal AJ. Epidemiology and natural history of nonalcoholic fatty liver Disease. Semin Liver Dis. 2015;35(3) 221-235.

3. Chalasani N, Younossi Z, Lavine JE, Charlton M, Cusi K, Rinella M, et al. The diagnosis and management of non alcoholic fatty liver disease: practice guideline by the American Association for the Study of Liver Diseases, American College of Gastroenterology, and the- American Gastroenterological Association. Hepatology. 2012.

4. Obika M, Noguchi H. Diagnosis and Evaluation of Nonalcoholic Fatty Liver
Disease. Experimental Diabetes Research. 2012

5. Argentou, M., Tiniakos, D. G., Karanikolas, M., Melachrinou, M., Makri, M. G., Kittas, C., \& Kalfarentzos, F. (2009). Adipokine Serum Levels Are Related to Liver Histology in Severely Obese Patients Undergoing Bariatric Surgery. Obesity Surgery, 19(9), 13131323. doi:10.1007/s11695-009-9912-9

6. Angulo P, Hui JM, Marchesini G, Bugianesi E, George J, Farrell GC, et al. The NAFLD fibrosis score: a noninvasive system that identifies liver fibrosis in patients with NAFLD. Hepatology. 2007;45(4):846-54

7. Kadowaki, T. (2006). Adiponectin and adiponectin receptors in insulin resistance, diabetes, and the metabolic syndrome. Journal of Clinical Investigation, 116(7), 1784-1792. doi:10.1172/jci29126

8. Polyzos SA, Toulis KA, Goulis DG, Zavos C, Kountouras J.Serut Total Adiponectin in nonalcoholic fatty liver disease : a systematic review and meta-analysis. Metabolism clinical an experimental. 2011; 60:313-326.

9. Pagano, C., Soardo, G., Pilon, C. et al. Increased Serum Resistin in Nonalcoholic Fatty Liver Disease is related to liver disease severity and not to insulin resistance. The Journal of Clinical Endocrinology and Mtabolism. 2005; 91(3): 1081-1086.

10. Balmer ML, Joneli J, Schoepfer A, Stickel F, Thormann W, Dufour JF. Signifcance of serum adiponectin levels in patients with chronic liver disease. Clin Sci (Lond) 2010; 119: 431-436

11. Da Silva TE, Costa-Silva M, Correa CG, Denardin G, Alencar MLA, Coelho MSPH, Muraro-Wildner L, Luiza-Bazzo M, González-Chica DA, Dantas-Correa EB, Narciso-Schiavon JL, Schiavon LL. Clinical Significance of Serum Adiponectin and Resistin Levels in Liver Cirrhosis. Ann Hepatol.2018; 17: 286-299

12. Hui JM, Sud A, Farrell GC, Bandara P, Byth K, Kench JG, et al. Insulin resistance is associated with chronic hepatitis $\mathrm{C}$ virus infection and fibrosis progression. Gastroenterology.2003;125:1695-1704

13. Yamauchi T, Kamon J, Ito Y, Tsuchida A, Yokomizo T, Kita S. Cloning of adiponectin receptors that mediate antidiabetic 
metabolic effects. Nature.2003; 423:762769.

14. Younossi ZM, Koenig AB, Abdelatif D, Fazel Y, Henry L, Wymer M. Global epidemiology of nonalcoholic fatty liver disease-Meta-analytic assessment of prevalence, incidence, and outcomes. Hepatology.2016; 64:73-84.

15. Perumpail, B. J., Khan, M. A., Yoo, E. R., Cholankeril, G., Kim, D., \& Ahmed, A.. Clinical epidemiology and disease burden of nonalcoholic fatty liver disease. World Journal of Gastroenterology. 2017; 23(47):8263-8276.

16. Wang, Y., Meng, R.-W., Kunutsor, S. K., Chowdhury, R., Yuan, J.-M., Koh, W.-P., \& Pan, A. (2018). Plasma adiponectin levels and type 2 diabetes risk: a nested casecontrol study in a Chinese population and an updated meta-analysis. Scientific Reports, 8(1). doi:10.1038/s41598-017-18709-9

17. Oikonomou, D., Georgiopoulos, G., Katsi, V., Kourek, C., Tsioufis, C., Alexopoulou, A. Tousoulis, D. Non-alcoholic fatty liver disease and hypertension. European Journal of Gastroenterology \& Hepatology, 30(9), 979-985. Pagano, C., Soardo, G., Esposito, W., Fallo, F., Basan, L., Donnini, D. Vettor, R. Plasma adiponectin is decreased in nonalcoholic fatty liver disease. European Journal of Endocrinology.2018;152(1):113118.

18. Kim, D. H., Kim, C., Ding, E. L., Townsend, M. K., \& Lipsitz, L. A. Adiponectin Levels and the Risk of Hypertension: A Systematic Review and Meta-Analysis. Hypertension. 2013;62(1): 27-32.

19. Angulo P, Hui JM, Marchesini G, Bugianesi E, George J, Farrell GC, et al. 2007. The NAFLD fibrosis score: a noninvasive system that identifies liver fibrosis in patients with NAFLD. Hepatology. 45(4): 846-54

20. McPherson S, Stewart SF, Henderson E, Burt AD, Day CP. Simple non-invasive fibrosis scoring systems can reliably exclude advanced fibrosis in patients with non-alcoholic fatty liver disease. Gut. 2010; 59(9):1265-1269.

How to cite this article: Andriani AD, Siregar GA, Lindarto D et al. The relationship of adiponectin level with non-alcoholic fatty liver disease (NAFLD) degrees. International Journal of Research and Review. 2021; 8(7): 199-206. DOI: https://doi.org/10.52403/ijrr. 20210727 